\title{
Clinical Implication of the Width of Subserosal Invasion in T3N0 Gastric Cancer
}

\author{
KENJI NAKAMURA ${ }^{1}$, MIFUJI TOMIOKU ${ }^{1}$, KAZUHITO NABESHIMA $^{1}$ and EIJI NOMURA ${ }^{2}$ \\ ${ }^{1}$ Department of Gastroenterological Surgery, \\ Tokai University School of Medicine, Isehara, Japan; \\ ${ }^{2}$ Department of Gastroenterological and General Surgery, \\ Tokai University Hachioji Hospital, Hachioji, Japan
}

\begin{abstract}
Background: The aim of this study was to clarify the impact of the horizontal width of tumor invasion into the subserosal layer on prognosis in patients with T3NO gastric cancer. Patients and Methods: A total of 72 patients with T3NO cancer were enrolled. Relapse-free survival of the subgroups classified according to width of subserosal invasion was compared to that of 34 patients with T4aNO cancer. Results: The group with wide invasion $(\geq 1.5 \mathrm{~cm})$ had a significantly poorer prognosis than those with narrow invasion $(<1.5 \mathrm{~cm})(p=0.014)$. Multivariate analysis revealed the width of subserosal invasion to be an independent prognostic factor. There was no significant difference between the T3NO group with wide invasion and the T4aNO group in the prognosis and recurrent status. Conclusion: The malignant potential of T3NO gastric cancer with wide subserosal invasion was found to be similar to that of T4aNO cancer.
\end{abstract}

In Japan, gastric cancer is treated according to the Japanese gastric cancer treatment guidelines 2014 (1). According to the guidelines, the standard treatment for curatively resectable advanced gastric cancer with invasion of the subserosa (T3) and no lymph node metastasis (N0) is surgery alone. However, a certain proportion of these patients develop recurrence, and nearly all patients with recurrence die of their disease. Therefore, it is considered to be of crucial clinical importance to identify the subset of patients with poor prognosis in this group.

This article is freely accessible online.

Correspondence to: Kenji Nakamura, MD, Department of Gastroenterological Surgery, Tokai University School of Medicine, 143 Shimokasuya, Isehara, Kanagawa 259-1193, Japan. Tel: +81 463931121, Fax: +81 463956491, e-mail: nakaken@is.icc.utokai.ac.jp

Key Words: Gastric cancer, subserosal invasion, prognosis.
Tumor diameter, lymphatic invasion, venous invasion, etc., have been considered as prognostic factors in patients with T3N0 gastric cancer (2-6). Opinions regarding the prognostic factors are divided, and no consensus has been reached. Therefore, in this study, we paid attention to the status of tumor invasion at the invasive front. Many studies of the status of tumor invasion at the invasive front have investigated changes in the serosal surface in patients with gastric cancer invading the serosa (7-10). However, there have been few studies investigating the status of tumor invasion into the subserosal layer in gastric cancer with invasion of the subserosa (11). Therefore, in the present study, we assessed the status of tumor invasion into the subserosal layer in T3N0 gastric cancer and investigated the possibility of its being a prognostic factor.

\section{Patients and Methods}

Patients. Between January 2006 and December 2015, 884 consecutive patients with gastric cancer underwent curative gastrectomy at the Department of Gastroenterological Surgery of Tokai University School of Medicine. Of these patients, the data of 72 in whom the histological examination revealed pT3N0 were analyzed in this retrospective study. The gross classification and histopathological classification were based on the Japanese Classification of Gastric Carcinoma published by the Japanese Gastric Cancer Association (JCGC) (12). Staging was performed according to the American Joint Committee on Cancer staging manual seventh edition (13). Patients were mainly followed-up on an outpatient basis at our hospital; however, those who had moved to other institutions were asked relevant questions by telephone. Follow-up was continued until June 2016, with a median duration of follow-up of 1574 days (range $=82-4373$ days). Diagnosis of tumor recurrence was based on clinical grounds. In patients with suspected recurrence, further investigations were performed. In some patients, the initial recurrence was diagnosed at two or more sites, and in such patients, all of the sites were counted as sites of initial recurrence.

Definition of width of subserosal invasion and clinicopathological parameters. The resected stomach was opened and placed on a flat board with the mucosal side up, and fixed in $10 \%$ formalin. After 
fixation, the neoplasm was sectioned along the maximum crosssectional plane parallel to the lesser curvature line, based on the general rules of the JCGC (12). Several additional sections parallel to the maximum cross-sectional plane and one section perpendicular to this plane were prepared to identify the area with the deepest invasion. For assessment of the status of tumor invasion into the subserosal layer, we examined the horizontal width of tumor invasion into the subserosal layer, that was measured according to the method described by Soga et al. (11); the horizontal distance measured along the outermost layer of the muscularis propria in these cross-sectional planes was defined as the horizontal width of subserosal invasion (Figure 1). When tumor invasion into the subserosal layer was observed in two or more sections, the maximum width was defined as the width of subserosal invasion in the patient.

The association between the width of subserosal invasion and clinicopathological parameters were evaluated, and the prognostic value of the width of subserosal invasion was assessed. In addition, the survival of the subgroups classified according to the width of subserosal invasion was compared to that of 34 patients with tumor penetrating the serosa but without lymph node metastasis (T4aN0).

Statistical analysis. The Chi-squared test was used to compare categorical data, and the Mann-Whitney $U$-or the Kruskal-Wallis Htest was used to compare continuous variables. Mean values were compared by the $t$-test. The cumulative relapse-free survival rates were calculated by the Kaplan-Meier method, and compared using the log-rank test. Multivariate analyses were performed to identify independent prognostic factors. All significant factors from the univariate analyses were entered in the multivariate analysis. The Cox proportional hazards regression model was used to determine the optimal cutoff threshold for width of subserosal invasion. Statistical significance was defined as $p<0.05$. Statistical calculations were performed with SPSS, version 22.0J (IBM Corp., Armonk, NY, USA).

\section{Results}

Threshold width of subserosal invasion. The median width of subserosal invasion in the patients with T3N0 gastric cancer was $0.98 \mathrm{~cm}$ (range $=0.03-3.87 \mathrm{~cm}$ ). In order to determine the relapse-free survival rates, changes in the subserosal width threshold were calculated at $0.5-\mathrm{cm}$ intervals. The optimal cutoff value was considered to correspond to the value associated with the largest HR as determined by the Cox proportional hazards regression model. The threshold value of $1.5 \mathrm{~cm}$ yielded the most significant difference in the survival rate $(\mathrm{HR}=3.272, p=0.020)$ (Table I).

Clinicopathologic characteristics according to the width of subserosal invasion. Of the 72 patients with T3N0 gastric cancer, 44 had a width of subserosal invasion of $<1.5 \mathrm{~cm}$ (narrow invasion group) and 28 had a width of subserosal invasion of $\geq 1.5 \mathrm{~cm}$ (wide invasion group). The clinicopathological factors were compared between these two groups (Table II). There were significant differences in the gross tumor type, with the infiltrative type being

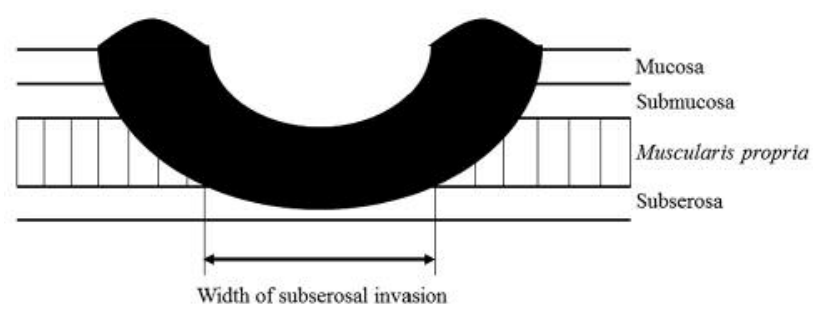

Figure 1. Methods of measurement of the horizontal width of subserosal invasion. Horizontal length of cancerous invasion into the subserosal layer was measured along the outermost layer of muscularis propria.

Table I. Hazard ratio (HR) according to the extent of subserosal invasion calculated by the Cox proportional hazards regression model using relapse-free survival.

\begin{tabular}{lccc}
\hline Threshold & HR & $95 \% \mathrm{CI}$ & $p$-Value \\
\hline $0.5 \mathrm{~cm}$ & 3.026 & $0.690-13.278$ & 0.142 \\
$1.0 \mathrm{~cm}$ & 2.759 & $0.969-7.852$ & 0.057 \\
$1.5 \mathrm{~cm}$ & 3.272 & $1.207-8.867$ & 0.020 \\
$2.0 \mathrm{~cm}$ & 2.114 & $0.803-5.564$ & 0.129 \\
$2.5 \mathrm{~cm}$ & 2.357 & $0.765-7.267$ & 0.136 \\
\hline
\end{tabular}

CI: Confidence interval.

Table II. Association between clinicopathological parameters and width of subserosal invasion in patients with T3NO gastric cancer.

\begin{tabular}{|c|c|c|c|c|c|}
\hline \multirow[t]{2}{*}{ Factor } & \multicolumn{2}{|c|}{ Narrow invasion } & \multicolumn{2}{|c|}{ Wide invasion } & \multirow[t]{2}{*}{$p$-Value } \\
\hline & $n=44$ & $(\%)$ & $\mathrm{n}=28$ & $(\%)$ & \\
\hline \multicolumn{6}{|l|}{ Age, years } \\
\hline$<65$ & 18 & $(40.9)$ & 14 & $(50.0)$ & 0.449 \\
\hline$\geq 65$ & 26 & $(59.1)$ & 14 & $(50.0)$ & \\
\hline \multicolumn{6}{|l|}{ Gender } \\
\hline Male & 31 & $(70.5)$ & 20 & $(71.4)$ & 0.929 \\
\hline Female & 13 & $(29.5)$ & 8 & $(28.6)$ & \\
\hline Tumor size, $\mathrm{cm}$ & 3.8 & & 4.6 & & 0.127 \\
\hline \multicolumn{6}{|l|}{ Gross type } \\
\hline Circumscribed & 34 & $(77.3)$ & 12 & $(42.9)$ & 0.003 \\
\hline Infiltrative & 10 & $(22.7)$ & 16 & $(57.1)$ & \\
\hline \multicolumn{6}{|l|}{ Histologic type } \\
\hline Differentiated & 25 & $(56.8)$ & 13 & $(46.4)$ & 0.389 \\
\hline Undifferentiated & 19 & $(43.2)$ & 15 & $(53.6)$ & \\
\hline \multicolumn{6}{|l|}{ Lymphatic invasion } \\
\hline Negative & 11 & $(25.0)$ & 4 & $(14.3)$ & 0.275 \\
\hline Positive & 33 & $(75.0)$ & 24 & $(85.7)$ & \\
\hline \multicolumn{6}{|l|}{ Venous invasion } \\
\hline Negative & 13 & $(29.5)$ & 5 & (17.9) & 0.264 \\
\hline Positive & 31 & $(70.5)$ & 23 & $(82.1)$ & \\
\hline
\end{tabular}

T3: Tumor invasion of subserosa, No: no lymph node metastasis, narrow invasion: width of subserosal invasion $<1.5 \mathrm{~cm}$, wide invasion: width of subserosal invasion $\geq 1.5 \mathrm{~cm}$. 
Table III. Univariate analysis of prognostic factors for the 5-year relapse-free survival (RFS) rate of patients with T3NO gastric cancer.

\begin{tabular}{lccc}
\hline & $\begin{array}{c}\text { Number of } \\
\text { patients }\end{array}$ & $\begin{array}{c}\text { 5-y RFS } \% \\
\text { Factor }\end{array}$ & $p$-Value \\
\hline Age, years & & & \\
$\quad<65$ & 32 & 72.8 & 0.137 \\
$\geq 65$ & 40 & 78.7 & \\
Gender & & & \\
$\quad$ Male & 51 & 79.8 & 0.549 \\
$\quad$ Female & 21 & 66.9 & \\
Tumor size, cm & & & \\
$\quad<7.0$ & 60 & 78.4 & 0.048 \\
$\quad \geq 7.0$ & 12 & 53.3 & \\
Gross type & & & \\
$\quad$ Circumscribed & 46 & 77.6 & 0.655 \\
$\quad$ Infiltrative & 26 & 73.6 & \\
Histologic type & & & \\
$\quad$ Differentiated & 38 & 81.5 & 0.355 \\
$\quad$ Undifferentiated & 34 & 70.2 & \\
Lymphatic invasion & & & \\
$\quad$ Negative & 15 & 87.5 & 0.126 \\
$\quad$ Positive & 57 & 72.9 & \\
Venous invasion & & & \\
$\quad$ Negative & 18 & 78.8 & 0.642 \\
$\quad$ Positive & 54 & 75.1 & \\
Width of subserosal invasion & & & \\
$\quad<1.5$ cm & 44 & 85.0 & 0.014 \\
$\quad \geq 1.5$ cm & 28 & 61.8 & \\
\hline
\end{tabular}

T3: Tumor invasion of subserosa, N0: no lymph node metastasis.

Table IV. Multivariate analysis of prognostic factors for the 5-year relapse-free survival (RFS) rate of patients with T3NO gastric cancer.

\begin{tabular}{lccc}
\hline Factor & HR & $95 \%$ CI & p-Value \\
\hline $\begin{array}{l}\text { Tumor size } \\
\quad<7.0 \mathrm{~cm}\end{array}$ & 2.173 & $0.596-7.926$ & 0.240 \\
$\quad \geq 7.0 \mathrm{~cm}$ & & & \\
Width of subserosal invasion & 3.267 & $1.203-8.870$ & 0.020 \\
$\quad<1.5 \mathrm{~cm}$ & & & \\
$\geq 1.5 \mathrm{~cm}$ & & & \\
\hline
\end{tabular}

T3: Tumor invasion of subserosa, N0: no lymph node metastasis, HR: hazard ratio, CI: confidence interval.

significantly more common in the wide invasion group than in the narrow invasion group $(p=0.003)$. However, there were no significant differences in tumor diameter, lymphatic invasion or venous invasion between the two groups.

Correlation of the width of subserosal invasion with prognosis. The prognostic impact of the width of subserosal invasion was evaluated by univariate and multivariate analyses. The 5-year

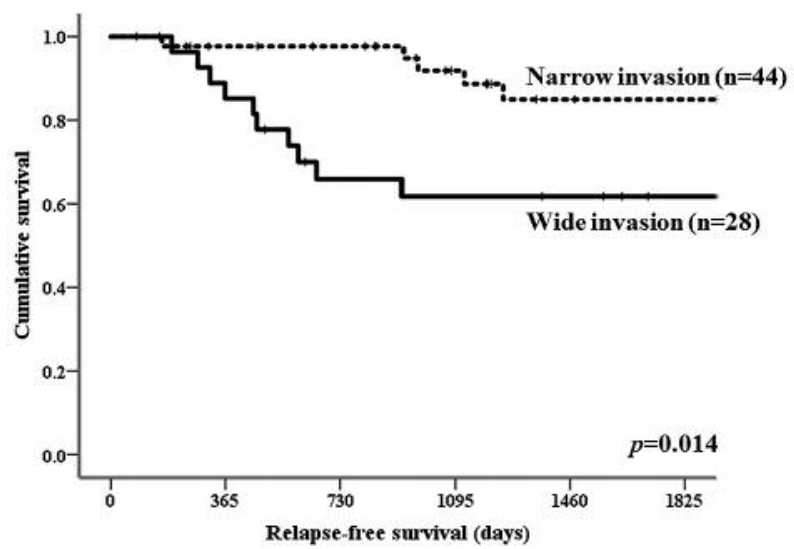

Figure 2. Kaplan-Meier survival curves for relapse-free survival of patients with T3NO gastric cancer according to width of subserosal invasion. The group with wide subserosal invasion had a significantly poorer prognosis compared to the group with narrow subserosal invasion. T3: Tumor invasion of subserosa, N0: no lymph node metastasis, narrow invasion: width of subserosal invasion $<1.5 \mathrm{~cm}$, wide invasion: width of subserosal invasion $\geq 1.5 \mathrm{~cm}$.

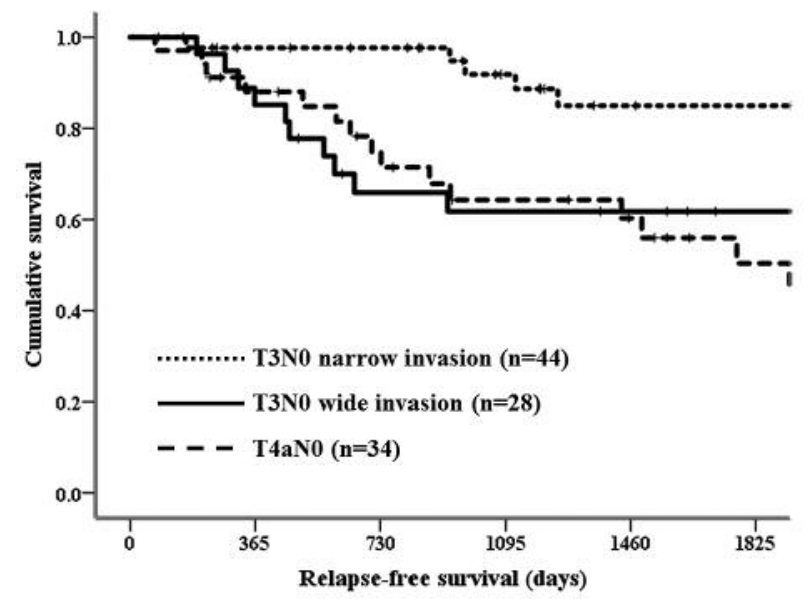

Figure 3. Kaplan-Meier survival curves for relapse-free survival of patients with gastric cancer. This figure shows the survival curves for patients with T3NO gastric cancer, divided according to the width of subserosal invasion, and compared to that of patients with T4aNO gastric cancer. There was no significant difference in survival curves for T3NO group with wide invasion and that for the T4aNO group $(p=0.582)$. T3: Tumor invasion of subserosa, NO: no lymph node metastasis, narrow invasion: width of subserosal invasion $<1.5 \mathrm{~cm}$, wide invasion: width of subserosal invasion $\geq 1.5 \mathrm{~cm}$, T4a: tumor penetration of serosa.

relapse-free survival rate was $85.0 \%$ in the narrow invasion group and $61.8 \%$ in the wide invasion group (Figure 2). The wide invasion group had a significantly poorer prognosis than did the narrow invasion group $(p=0.014)$. The only other prognostic factor for which a significant difference was found 
in vivo $31: 409-413(2017)$

Table V. Comparison of recurrence patterns between patients with T3NO with different width of subserosal invasion and patients with T4aNO gastric cancer.

\begin{tabular}{|c|c|c|c|c|c|c|c|c|c|}
\hline & \multicolumn{4}{|c|}{ T3NO } & \multicolumn{2}{|c|}{ T4aN0 } & \multicolumn{3}{|c|}{$p$-Value } \\
\hline & \multicolumn{2}{|c|}{ (A)Narrow } & \multicolumn{2}{|c|}{ (B)Wide } & \multirow[b]{2}{*}{$\mathrm{n}=34$} & \multirow[b]{2}{*}{$(\%)$} & \multirow[b]{2}{*}{ (A) $v s$. (B) } & \multirow[b]{2}{*}{ (B) $v s . \mathrm{T} 4 \mathrm{aN} 0$} & \multirow[b]{2}{*}{ (A) $v s . \mathrm{T} 4 \mathrm{aNO}$} \\
\hline & $\mathrm{n}=44$ & $(\%)$ & $\mathrm{n}=28$ & $(\%)$ & & & & & \\
\hline Recurrence & 4 & $(9.1)$ & 9 & $(32.1)$ & 10 & $(29.4)$ & 0.013 & 0.816 & 0.009 \\
\hline \multicolumn{10}{|l|}{ Site } \\
\hline Peritoneal & 1 & $(25.0)$ & 5 & $(55.6)$ & 6 & $(60.0)$ & & & \\
\hline Hematogenous & 3 & $(75.0)$ & 3 & (33.3) & 3 & $(30.0)$ & & & \\
\hline Lymph node & 0 & & 1 & $(11.1)$ & 1 & $(10.0)$ & & & \\
\hline
\end{tabular}

T3: Tumor invasion of subserosa, No: no lymph node metastasis, T4a: tumor penetration of serosa, narrow invasion: width of subserosal invasion $<1.5 \mathrm{~cm}$, wide invasion: width of subserosal invasion $\geq 1.5 \mathrm{~cm}$.

between the two groups by univariate analysis was the tumor size $(p=0.048)$; there were no significant differences in the mean age, gross type, histological type, lymphatic invasion, or venous invasion (Table III). Multivariate analysis identified the width of subserosal invasion as the sole independent prognostic factor (hazard ratio $=3.267, p=0.020$ ) (Table IV).

Comparison with T4aNO gastric cancer. The survival of the subgroups with $\mathrm{T} 3 \mathrm{~N} 0$ gastric cancer classified according to the width of subserosal invasion was compared to that of the 34 patients with T4aN0 gastric cancer. The 5-year relapse-free survival rate in the patients with T4aN0 gastric cancer was $50.4 \%$, significantly lower than that in patients with T3N0 gastric cancer with narrow subserosal invasion of $85.0 \%$ $(p=0.001)$ (Figure 3). However, there was no significant difference between the patients of the T4aN0 group and the T3N0 group with wide subserosal invasion $(p=0.582)$.

The recurrence rate was $9.1 \%(4 / 44)$ in the T3N0 group with narrow subserosal invasion, $32.1 \%(9 / 28)$ in the T3N0 group with wide subserosal invasion, and $29.4 \%$ (10/34) in the T4aN0 group (Table V). The recurrence rate was significantly higher in the T3N0 group with wide invasion and the T4aN0 group than in the T3N0 group with narrow invasion $(p=0.013$ and $p=0.009$, respectively). Peritoneal metastasis accounted for the majority of recurrences in both the T3N0 group with wide invasion and the T4aNO group. The time interval between surgery and recurrence was 2 years 7 months in the T3N0 group with narrow invasion, 1 year 3 months in the T3N0 group with wide invasion, and 1 year 10 months in the T4aN0 group.

\section{Discussion}

In Japan, the use of adjuvant chemotherapy after gastric cancer surgery is in accordance with the Japanese gastric cancer treatment guidelines 2014 (1). According to the guidelines, adjuvant chemotherapy is not indicated for patients with T3N0 gastric cancer who have undergone curative resection, even though some of these patients present with recurrence. Therefore, it might be useful to identify the subset of patients with $\mathrm{T} 3 \mathrm{~N} 0$ gastric cancer who are at a high risk for the development of recurrence, and who might benefit from adjuvant chemotherapy.

Many studies of the status of tumor invasion at the invasive front have analyzed the changes in the serosal surface in gastric cancer invading the serosa (14-16). In one such study, the maximum diameter of the region with altered serosal surface was measured (16), and in another, the status of alteration of the serosal surface was classified according to macroscopic findings (15). The pattern of tumor infiltration into the surrounding tissue was investigated in serosa-negative advanced gastric cancer i.e. muscularis propria- or subserosainvading tumor. The pattern is defined by the JCGC, which categorizes gastric cancer as expansive growth type, infiltrative growth type, and intermediate type (12). Song et al. reported that this pattern type was associated with the poorer prognosis in patients with T3 gastric cancer (17). Saito et al. reported that lymph node involvement, but not the pattern type, is a prognostic factor in T3 gastric cancer (18). The report by Soga et al. is the only recent study of the horizontal width of tumor invasion into the subserosal layer in T3 gastric cancer (11). However, in their report, $65 \%$ of their patients also had lymph node metastasis. Therefore, there have been no recent studies on the width of tumor invasion into the subserosal layer in patients with T3N0 gastric cancer.

In the present study, the cut-off width of tumor invasion into the subserosal layer for discriminating patients with a poor prognosis was determined to be $1.5 \mathrm{~cm}$. Soga et al. conducted a similar study but determined that the optimal cutoff value was $2.0 \mathrm{~cm}$ (11). In their study, $65 \%$ of the patients had lymph node metastasis, and the median tumor diameter was $5.0 \mathrm{~cm}$. In the present study, none of the patients had lymph node metastasis, and the median tumor diameter was $4.2 \mathrm{~cm}$, suggesting that the difference in the cutoff values used in the two studies was caused by differences in the characteristics of the study populations. 
The prognosis was significantly poorer in patients with wide subserosal invasion than in those with narrow invasion. Multivariate analysis identified the width of invasion as an independent prognostic factor. In regard to the cause of poor prognosis in patients with wide invasion, Soga et al. hypothesized that tumors with wide tumor invasion into the subserosal layer were more likely to have latent serosal invasion in unsectioned planes (11). However, the above findings may also be true for patients with narrow tumor invasion. In the present study, we also measured the distance from the outermost layer of the muscularis propria to the deepest part of the tumor invading the subserosal layer (vertical length) (data not shown). There was a significant difference in the median vertical length between the narrow and wide invasion groups $(0.7$ and $2.3 \mathrm{~mm}$, respectively; $p=0.001)$. Thus, the vertical length in the subserosal layer was significantly greater in the group with wide invasion, suggesting that the tumor burden in this layer was greater than that in the group with narrow invasion. The difference in prognosis between the two groups was considered to be caused by the difference in the tumor burden.

There was no significant difference in the prognosis between the T3N0 gastric cancer group with wide subserosal invasion and the T4aN0 group. Nor were there any differences in the recurrence rate between the two groups. Furthermore, peritoneal metastasis accounted for the majority of recurrences in both groups, and the period to recurrence was less than 2 years in both groups. These results suggest that the malignant potential of tumors with wide subserosal invasion in T3N0 gastric cancer is similar to that of $\mathrm{T} 4 \mathrm{aN} 0$ gastric cancer.

In conclusion, the present study showed that the horizontal width of cancer invasion of the subserosal layer was an independent predictor of relapse-free survival in patients with T3N0 gastric cancer. The prognosis and recurrent status of T3N0 cancer with a wide subserosal invasion was similar to that of T4aN0 gastric cancer. In the assessment of patients with $\mathrm{T} 3 \mathrm{~N} 0$, therefore, it is important to classify the patients based on the horizontal width of subserosal invasion into the narrow invasion group and wide invasion group.

\section{References}

1 Japanese Gastric Cancer Association: Japanese gastric cancer treatment guidelines 2014 (ver.4). Gastric Cancer 20: 1-19, 2017.

2 Lee IS, Yook JH, Kim TH, Kim HS, Kim KC, Oh ST and Kim BS: Prognostic factors and recurrence pattern in node-negative advanced gastric cancer. Eur J Surg Oncol 39: 136-140, 2013.

3 Huang CM, Wang HM, Zheng CH, Li P, Xie JW, Wang JB, Lin $\mathrm{JX}$ and $\mathrm{Lu} \mathrm{J}$ : Tumor size as a prognostic factor in patients with node-negative gastric cancer invading the muscularis propria and subserosa (pT2-3N0M0 stage). Hepato-Gastroenterol 60: 699-703, 2013.

4 Araki I, Hosoda K, Yamashita K, Katada N, Sakuramoto S, Moriya $\mathrm{H}$, Mieno $\mathrm{H}$, Ema A, Kikuchi S, Mikami $\mathrm{T}$ and Watanabe M: Prognostic impact of venous invasion in stage IB node-negative gastric cancer. Gastric Cancer 18: 297-305, 2015.
5 Imamura T, Komatsu S, Ichikawa D, Kubota T, Okamoto K, Konishi H, Shiozaki A, Fujiwara H, Morimura R, Murayama Y, Kuriu Y, Ikoma H, Nakanishi M, Sakakura C and Otsuji E: Poor prognostic sub group in T3N0 stage IIA gastric cancer, suggesting an indication for adjuvant chemotherapy. J Surg Oncol 111: 221-225, 2015.

6 Saito H, Murakami Y, Miyatani K, Kuroda H, Matsunaga T, Fukumoto Y, Osaki T and Ikeguchi M: Predictive factors for recurrence in $\mathrm{T} 2 \mathrm{NO}$ and $\mathrm{T} 3 \mathrm{~N} 0$ gastric cancer patients. Langenbecks Arch Surg 401: 823-828, 2016.

7 Abe S, Shiraishi M, Nagaoka S, Yoshimura H, Dhar DK and Nakamura T: Serosal invasion as the single prognostic indicator in stage IIIA (T3N1M0) gastric cancer. Surgery 109: 582-588, 1991.

8 Bando E, Kawamura T, Kinoshita K, Takahashi S, Maeda A, Osada S, Tsubosa Y, Yamaguchi S, Uesaka K and Yonemura Y: Magnitude of serosal changes predicts peritoneal recurrence of gastric cancer. J Am Coll Surg 197: 212-222, 2003.

9 Yasuda K, Shiraishi N, Imomata M, Shiroshita H, Izumi K and Kitano S: Prognostic significance of macroscopic serosal invasion in advanced gastric cancer. Hepato-Gastroenterol 54: 2028-2031, 2007.

10 Yoo C, Ryu MH, Park YS, Yoo MW, Park SR, Ryoo BY, Jang SJ, Yook JH, Kim BS and Kang YK: Intraoperatively assessed macroscopic serosal changes in patients with curatively resected advanced gastric cancer: clinical implications for prognosis and peritoneal recurrence. Ann Surg Oncol 22: 2940-2947, 2015.

11 Soga K, Ichikawa D, Yasukawa S, Kubota T, Kikuchi S, Fujiwara H, Okamoto K, Ochiai T, Sakakura C, Kokuba Y, Yanagisawa A and Otsuji E: Prognostic impact of the width of subserosal invasion in gastric cancer invading the subserosal layer. Surgery 147: 197-203, 2010.

12 Japanese Gastric Cancer Association: Japanese classification of gastric carcinoma: 3rd English edition. Gastric Cancer 14: 101$112,2011$.

13 Edge SB and Compton CC: The American Joint Committee on Cancer: the 7th edition of the AJCC cancer staging manual and the future of TNM. Ann Surg Oncol 17: 1471-1474, 2010.

14 Baba H, Korenaga D, Haraguchi M, Okamura T, Saito A, Watanabe A and Sugimachi K: Width of serosal invasion and prognosis in advanced human gastric cancer with special reference to the mode of tumor invasion. Cancer 64: 2482-2486, 1989.

15 Sun Z, Xu YY, Wang ZN, Zhu Z, Zhang H, Huang BJ, Xu Y, Chen JQ and $\mathrm{Xu}$ HM: Macroscopic serosal classification predicts peritoneal recurrence for patients with gastric cancer underwent potentially curative surgery. Ann Surg Oncol 18: 1068-1080, 2011.

16 Kang Y, Li S, Ge Q, Liu X, Yang Z, Xue Y and Wang F: Extent of serosal changes predicts peritoneal recurrence and poor prognosis after curative surgery for gastric cancer. Medicine 94: e1750, 2015.

17 Song KY, Hur H, Jung CK, Jung ES, Kim SN, Jeon HM and Park $\mathrm{CH}$ : Impact of tumor infiltration pattern into the surrounding tissue on prognosis of the subserosal gastric cancer (pT2b). Eur J Surg Oncol 36: 563-567, 2010.

18 Saito H, Miyatani K, Takaya S, Kuroda H, Matsunaga T, Fukumoto Y, Osaki T and Ikeguchi M: Tumor infiltration pattern into the surrounding tissue has prognostic significance in advanced gastric cancer. Virchows Arch 467: 519-523, 2015.

Received February 2, 2017

Revised March 17, 2017

Accepted March 23, 2017 